\title{
Development of an invasive brain-machine interface with a monkey model
}

\author{
ZHANG QiaoSheng ${ }^{1,3,4}$, ZHANG ShaoMin ${ }^{1,3,4}$, HAO YaoYao ${ }^{1,3,4}$, ZHANG HuaiJian ${ }^{1,3,4}$, \\ ZHU JunMing ${ }^{1,5}$, ZHAO Ting ${ }^{1}$, ZHANG JianMin ${ }^{5}$, WANG YiWen ${ }^{1}$, ZHENG XiaoXiang ${ }^{1,3,4}$ \& \\ CHEN WeiDong ${ }^{1,2^{*}}$
}

${ }^{1}$ Qiushi Academy for Advanced Studies, Zhejiang University, Hangzhou 310027, China;

${ }^{2}$ College of Computer Science and Technology, Zhejiang University, Hangzhou 310027, China;

${ }^{3}$ Department of Biomedical Engineering, Zhejiang University, Hangzhou 310027, China;

${ }^{4}$ Key Laboratory of Biomedical Engineering of Ministry of Education, Zhejiang University, Hangzhou 310027, China;

${ }^{5}$ Department of Neurosurgery, Second Affiliated Hospital School of Medicine, Zhejiang University, Hangzhou 310009, China

Received October 12, 2011; accepted January 19, 2012

\begin{abstract}
Brain-machine interfaces (BMIs) translate neural activities of the brain into specific instructions that can be carried out by external devices. BMIs have the potential to restore or augment motor functions of paralyzed patients suffering from spinal cord damage. The neural activities have been used to predict the 2D or 3D movement trajectory of monkey's arm or hand in many studies. However, there are few studies on decoding the wrist movement from neural activities in center-out paradigm. The present study developed an invasive BMI system with a monkey model using a 10×10-microelectrode array in the primary motor cortex. The monkey was trained to perform a two-dimensional forelimb wrist movement paradigm where neural activities and movement signals were simultaneous recorded. Results showed that neuronal firing rates highly correlated with forelimb wrist movement; > $70 \%$ (105/149) neurons exhibited specific firing changes during movement and $>36 \%$ (54/149) neurons were used to discriminate directional pairs. The neuronal firing rates were also used to predict the wrist moving directions and continuous trajectories of the forelimb wrist. The four directions could be classified with $96 \%$ accuracy using a support vector machine, and the correlation coefficients of trajectory prediction using a general regression neural network were above 0.8 for both horizontal and vertical directions. Results showed that this BMI system could predict monkey wrist movements in high accuracy through the use of neuronal firing information.
\end{abstract}

brain-machine interface, primary motor cortex, center-out paradigm, neural decoding, support vector machine, general regression neural network

Citation: Zhang Q S, Zhang S M, Hao Y Y, et al. Development of an invasive brain-machine interface with a monkey model. Chin Sci Bull, 2012, 57: 2036-2045, doi: $10.1007 / \mathrm{s} 11434-012-5096-0$

It is demonstrated that brain-machine interfaces (BMIs) can transform motor intention into actions by decoding neural activities recorded from the brain [1-6]. This emerging field has been inspired by the requirements of restoring motor functions for individuals with severe motor deficits [7,8]. A typical BMI system consists of three parts, including a sensor, a decoder and an actuator [9]. The sensor records neural

*Corresponding author (email: qaas@ zju.edu.cn) activities that correspond to brain-desired actions [10-12]. The decoder converts the recorded neural signals into kinematics parameters for controlling the actuator, which could be a prosthetic arm or a screen cursor [13,14].

BMI systems can be divided into non-invasive or invasive ones with respect to the type of physiological signals [15]. Non-invasive BMIs measure synchronous activities of thousands of cortical neurons, such as electroencephalogram (EEG) activities, via electrodes positioned on the 
scalp [16-19]. By contrast, invasive BMIs typically use microelectrodes implanted in the cortex to record extracellular activities of neurons [20-23]. Since the past decade, we have witnessed exciting progress of invasive BMIs, which have been used to control devices with a much higher degree of freedom compared to the non-invasive ones [24-26]. However, because many of the technical complications of invasive BMI systems have not been solved, such as sensor safety, stability of recorded signals and decoding accuracy, further studies are needed for the development of invasive BMI systems that are effective for human beings [27,28].

To take on the challenge, it is better to conduct extensive researches on animal-based BMI platforms due to irreversible damages caused by current invasive recording techniques. In recent years, non-human primates (NHP) have been considered as appropriate subjects for invasive BMI studies for two reasons. First, the functional brain structure of NHPs is similar to that of humans [29]. Second, monkeys can be trained to perform and achieve more complicated operant tasks than other animal models, which are critical for obtaining a vast amount of neural information corresponding to complex motor behaviors [30-32].

In BMI studies, monkeys are often trained in behavioral tasks to control effector motions, such as free-limb movements or joystick operation [33]. In the 1980s, Georgopoulos et al. [34,35] constructed a standard center-out task, in which monkeys were trained to move a lightweight, frictionless joystick to capture one in eight randomly lighted targets to obtain a liquid reward. This paradigm has been improved and employed in many BMI studies to research forelimb movement in later 30 years [36,37]. Some other paradigms are also developed for decoding the movement trajectory of monkey arm, hand or finger [38]. For example, monkeys were trained to make one-dimensional hand movements to displace a joystick in one of two directions [26]. Monkeys have also been trained to move an arm in a 3D virtual environment [39]. However, few studies have focused on the wrist movement research in a center-out paradigm [40].

Recent results have revealed that cortical neurons contribute to every aspect of movements [41], and many algorithms have been successfully used to decode limb movement with neuronal firing rates in animal models. For instance, linear regression and artificial neural networks have been used to predict real-time hand position [26], and a linear filter has been used to reconstruct hand trajectory by regressing both position and velocity [27]. A modified population vector algorithm performs well in decoding $3 \mathrm{D}$ hand movement parameters [39], and reach direction and grasp type can be extracted from multi-unit activities using support vector machine algorithms [42]. In addition, finger movement has been decoded using a hierarchical classification method [40].

In this paper, we presented a monkey training system based on the center-out task for decoding wrist movement, which had been relatively ignored in previous studies. In this system, the subject monkey moved a joystick to perform a four-direction center-out task with its wrist. During the task, the two-dimensional positions of joystick were synchronously recorded with 96-channel neuronal signals. We evaluated the correlation between the neuronal firing rates and the forelimb wrist movement. Two decoding algorithms were employed to predict the forelimb wrist movement direction and continuous trajectory from the population activities of the recorded neurons.

\section{Experiments and methods}

\subsection{Training paradigm and system construction}

In this paper, a male rhesus monkey (Macaca mulatta), labeled as B01, was trained to perform four direction center-out task by turning a joystick to move a cursor on a visual display from the center location to one of the four directions (up, down, left, right) to hit the target. This task reflected the kinematics of the wrist movement. In order to train the monkey, a monkey behavior training system was developed in this study [43], as shown in Figure 1. In this system, the monkey was first trained to sit on a primate chair and grasp a joystick with his hand. A monitor was situated about $100 \mathrm{~cm}$ in front of the monkey's face. The monkey could comfortably see the monitor screen without turning his head. Two circles were presented on the screen. The larger circle served as the visual target, which randomly appeared in any position of four directions. The smaller circle, which was initially shown in the center of the monitor, indicated joystick position. During the experiment, the monkey held the joystick and moved it across the two-dimensional $x-y$ plane to guide the smaller circle to the

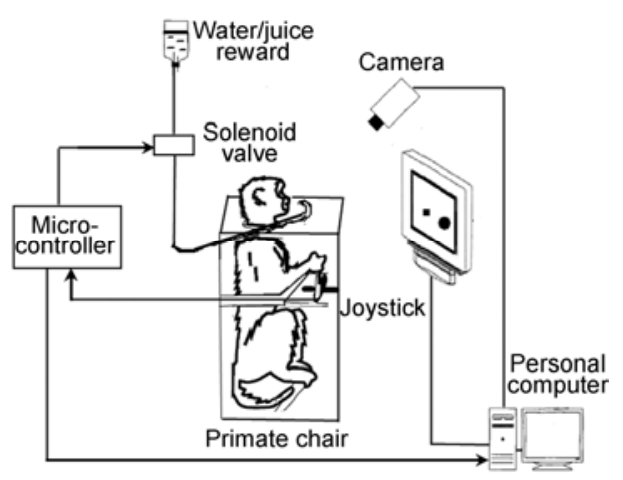

Figure 1 Schematic diagram of the primate behavioral training system. Monkey learned to control the joystick with his wrist. The position of the joystick was transformed to be the small cursor of the monitor. The monkey was trained to move the small cursor to bigger visual target circle. The whole training process was monitored by the infrared camera. If the monkey successfully hit the target within predefined time range, personal computer (PC) would send a message to micro-controller unit (MCU). And the MCU opened the solenoid valve to give the water or juice drops as reward. All these PC control programs were written in Visual C++ 6.0. 
target. When the two circles overlapped, the color of the two circles was changed, which indicated that the trial was successful, and the monkey received some water reward. During experimentation, a micro-controller unit (MCU) recorded joystick positions, which represented wrist trajectory. In our study, B01 was trained to perform this task until achieving a success rate $>95 \%$ prior to implantation surgery. All the experimental procedures described in this section and the following section (1.2) were followed the Guide for the Care and Use of Laboratory Animals (Ministry of Health of the People's Republic of China).

\subsection{Surgery procedures and data acquisition}

Following training, B01 was implanted with a 100-electrode Utah array $(1.0 \mathrm{~mm}$ electrode length; $7.0 \mathrm{~cm}$ wire bundle length; ICS-96 Connector, Blackrock Microsystems, Salt Lake City, UT, USA) in the primary motor cortex (M1) of the cerebral hemisphere contralateral to the hand performing the task [43], as shown in Figure 2(a). In addition, two head posts were placed on the skull for head stabilization during neural recording and array pedestal fix, respectively. The surgery was performed under standard sterile conditions and general anesthesia by following previously described methods, with appropriate modifications [44]. The monkey was sedated with ketamine $(10 \mathrm{mg} / \mathrm{kg})$ and diazepam $(1 \mathrm{mg} / \mathrm{kg})$. A stable level of deep anesthesia was maintained via endotracheal administration of isoflurane (1\%$2 \%$ ) with a veterinary anesthesia ventilator (Matrx VME2, Midmark, Orchard Park, NY, USA) for the entire surgical procedure. After fixing the head in a standard stereotaxic frame (51801, Stoelting, Wood Dale, IL, USA), we exposed the skull via a skin incision, with povidone iodine applied for disinfection. Two head posts were fixed to the skull with titanium screws. The array connector was fixed on one head post by a white pedestal, as shown in Figure 2(b). Then a craniotomy was performed over the motor cortex through a rectangular cranial opening. The dura was incised and the array was placed above the primary motor cortex, as shown in Figure 2(a). The shape of the wire bundle was adjusted until the tips of electrode array were perpendicular to the
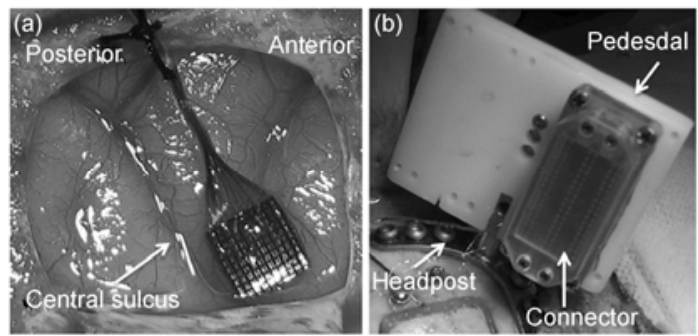

Figure 2 Array location and array connector. (a) Craniotomy site and array location. A Utah array was implanted in the wrist area of the primary motor cortex. (b) ICS-96 connector of Utah array and the fixing pedestal. The connector was attached to the pedestal by screws. The pedestal was fixed to the head post, which was attached to the head with titanium screws. cortical surface. The array was then quickly inserted into the motor cortex using a pneumatic insertion device (Micro Implantable Systems, Salt Lake City, UT, USA). The cortical surface and array were covered with a sheet of sterile DuraGen, and the dura was replaced above the array and sutured in place after reference wires were inserted in the subdural space. The second piece of DruaGen was placed over the dura, and the bone flap was replaced over the cranial defect and fixed in place using titanium plates screwed to the skull. Finally, fascia and skin were stitched around the connector. During the entire surgical procedure, continuous heart rate, expired end tidal carbon dioxide $\left(\mathrm{ETCO}_{2}\right)$, breathing rate, blood pressure and oxygen saturation were monitored with a physiological monitor (BeneView T5, Mindray, Shenzhen, China). The body temperature was maintained at $37^{\circ} \mathrm{C}$ with a heating pad (T/PUMP, Gaymar, Orchard Park, NY, USA).

The monkey was allowed to recover from surgery for at least one week. Antibiotic therapy (Ceftriaxone sodium, 1 $\mathrm{g} / \mathrm{d}$ ) was continuously injected for $5 \mathrm{~d}$. After recovery, the monkey started to perform center-out tasks. Behavioral tasks were performed twice daily (morning and afternoon), and each experiment lasted approximately $60 \mathrm{~min}$. Multi-channel neural signals and joystick positions were synchronously recorded during behavior tasks, and neural signals were recorded using a Cerebus $128^{\mathrm{TM}}$ (Blackrock Microsystems, Salt Lake City, UT, USA) with a 30-kHz sampling rate. Each neural signal channel was initially filtered using a third-order analog Butterworth band-pass filter from 0.3 to $7500 \mathrm{~Hz}$, followed by filtration through a fourth-order digital Butterworth high-pass filter with a cutting frequency of $250 \mathrm{~Hz}$. Neuronal spikes were detected using the threshold crossing method, in which the threshold was set to -6.25 times the signal baseline root mean square (RMS) of all channels to detect the negative peak of spike waveforms. Two-dimensional joystick positions were recorded with a MCU system at a $20-\mathrm{Hz}$ sampling rate. The multi-channel neural data and joystick positions were synchronized for further analysis.

\subsection{Data analysis}

In our study, only well performed behavioral tasks were selected for off-line data analysis. Experiments with a low targeting success rate, turbid joystick data, or noisy neural data were not considered. To obtain single neuronal activities, spikes recorded from each channel were sorted offline by K-means clustering. Neuronal firing rates were calculated by counting spike numbers in each 100-ms window (bin). Two-dimensional joystick data were also resampled in a $100-\mathrm{ms}$ window by averaging between two points. The correlation between neuronal firing rates and joystick moving direction was analyzed.

In the direction decoding section, a support vector machine (SVM, by LIBSVM packet, library for support vector 
machine) was used to predict moving directions [45]. The SVM parameters consisted of $\mathrm{C}$ (regularization parameter) and Gamma (spreading parameter in radical basis function), which were set to 2 and 0.3125 respectively. The values were found by grid search, in which the two parameters were discretized and every possible combination of them was evaluated.

The continuous wrist movement trajectory was predicted with the general regression neural network (GRNN) method based on the probability density estimation of the observed data $[46,47]$. For the GRNN decoding model in our application, the inputs were the neuronal firing rates, and model outputs were the predicted joystick positions. In a single recording session, the data was separated into several identical segments. In each data segment, half of the data was used to train the GRNN model, the other half was used to test the model. The GRNN spreading parameter was set to 2.5 in all data segments. This value was determined by searching the best prediction accuracy in five-fold cross validation on the training dataset. Decoding performance was evaluated by Pearson's correlation coefficient (CC) and mean square error (MSE).

\section{Results}

\subsection{Long-term variation of recorded neuronal number}

Neuronal activities had been recorded for more than seven months and the number of recorded neurons over time was shown in Figure 3. After spike sorting, a total of 207 neurons were detected from all electrodes one week after implantation surgery. The number of neurons had decreased until one month later. This number kept around 80 during the following five months (Figure 3). The color maps in Figure 3 depicted neuronal numbers at each electrode. Some neurons at specific electrodes, such as N78, N68, and N19, disappeared over time, and some neurons that were not previously identified showed up in some other electrodes, such as N96, N98, and N99. These results demonstrated that the total neuron number stabilized after the gradual loss of neuronal signals during the first month of recording. These results were consistent with previously published results $[11,44,48]$.

\subsection{Correlation between joystick movement direction and neuronal firing rates}

Data recorded in the afternoon of November 19, 2010 (120 d after surgery) served as the offline analysis example in this paper. Four direction targets were randomly distributed, as shown in Figure 4(a), and two-dimensional joystick positions detected within $150 \mathrm{~s}$ were presented with $x$-axis and $y$-axis data, respectively. These results suggested that the monkey could perform the task well. A total of 149 neurons were detected in the dataset, in which a diversity of firing rates was observed. Some neurons exhibited very sparse firing, which some others fired much more frequently.

To determine the capacity of discriminating joystick moving direction with population of neuronal firing rates, both neuronal firing rates and joystick position data were extracted from continuous recordings around moving events. Positive and negative thresholds were set to detect leftmoving and right-moving events in the $x$-axis of joystick data. Similarly, two thresholds were set in the $y$-axis to extract up-moving and down-moving events. A 3-s segment of data, which starts at $0.7 \mathrm{~s}$ before the onset, was selected for each moving trial. Moving onsets were defined as the timeline origin $(0 \mathrm{~s})$ for all trials. A total of 159 left-moving events, 199 right-moving events, 122 up-moving events, and 84 down-moving events were extracted, as shown in Figure 5(b). Results demonstrated that moving trials exhibited different durations, which suggested that firing patterns could be different at the end of trials. Therefore, only time windows around moving onsets were used for further analysis. For each moving direction, neuronal firing rates were averaged across all trials. Subsequently, to determine

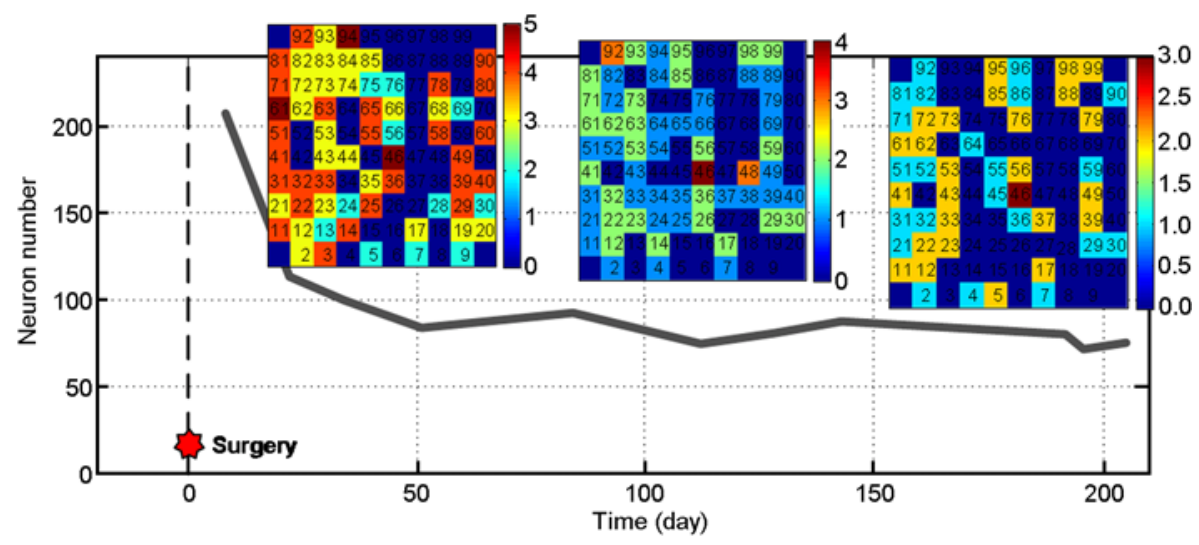

Figure 3 Long-term neuronal number trends. Surgery was performed on day 0. Neurons from each electrode were extracted using the threshold cross method and were sorted by K-means clustering. The color map indicated spatial position of electrodes and related neuronal numbers. 


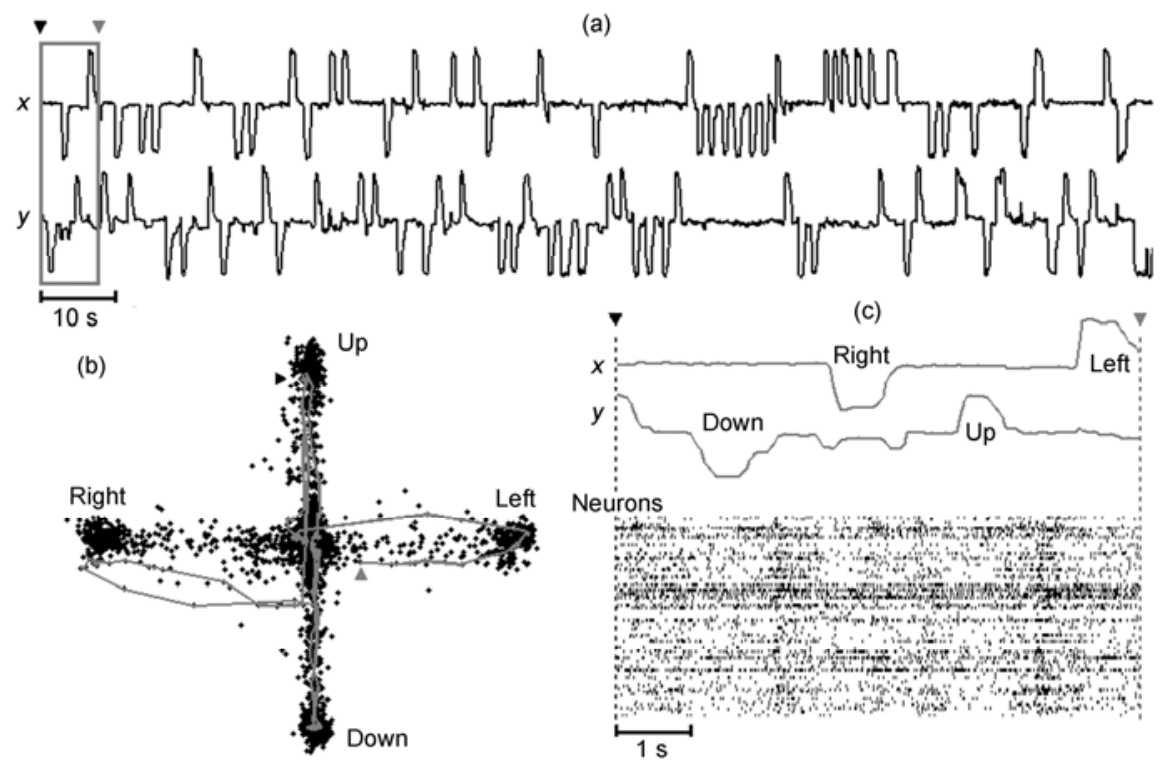

Figure 4 Synchronously recorded two-dimensional joystick data and population level neuronal firings. (a) Two-dimensional $x$-y joystick position within 150 seconds; (b) black dots indicated the identical $150 \mathrm{~s}$ combined $x-y$ joystick position. Grey line represented the joystick trajectory. Black and grey triangles represented trajectory beginning and end, respectively. (c) Population level neuronal firing raster during four-directional joystick movement was represented. The top grey lines represented the $x-y$ joystick position within the time range of the grey rectangle in (a). The bottom short line indicated the neuronal spike firing raster. The vertical axis represented neurons extracted from electrodes, and the horizontal axis represented the timeline.
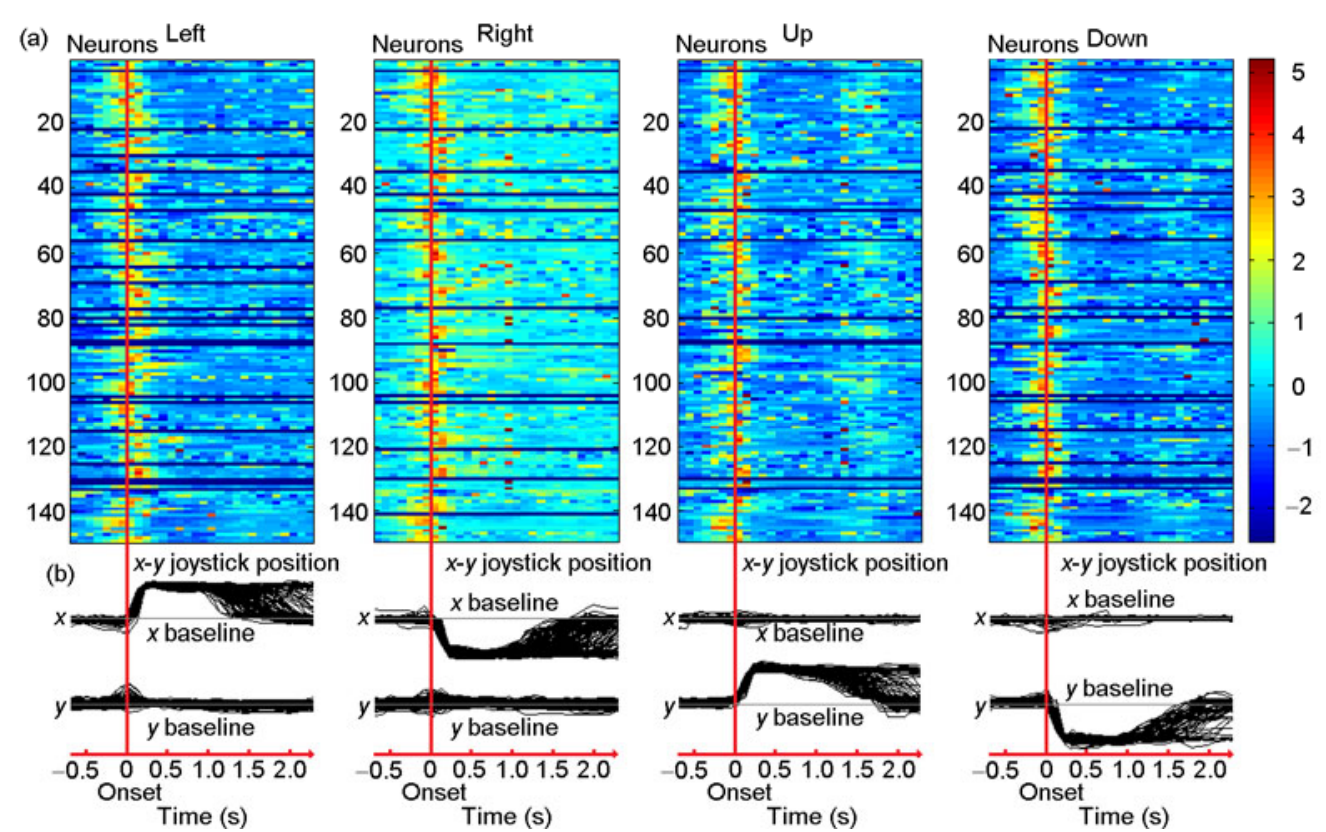

Figure 5 Joystick movement trials and corresponding neuronal firing rates. (a) Averaged neuronal firing rates from four directions. The horizontal axis represented the timeline and the vertical axis indicated neuronal counts. Neuronal firing rates were computed in a 100-ms window without overlapping. Firing rates for each neuron were normalized across time. (b) Joystick movement trials in four directions. Onsets of moving events were defined as origin of timeline $(0 \mathrm{~s})$. Grey lines indicated baseline of joystick position and red lines indicated moving onsets. Joystick movement trials were extracted via thresholding methods in two-dimensional position recordings.

the correlation between neuronal firing rates and the joystick moving direction, each neuron was normalized by subtracting the mean value and dividing by the standard deviation across the timeline. Neuronal firing rates from all four directions were shown in Figure 5(a). The results demonstrated that neuronal firing rates increased at 200$300 \mathrm{~ms}$ prior to moving onsets, but decreased to baseline levels at 200-300 ms after moving onsets.

To identify neurons with significantly different firing rates before and during moving onsets across the timeline, 
one-way ANOVA (analysis of variance) was used for analysis at each moving direction. A total of seven bins prior to the onset of joystick movement, as well as four bins after the onset, were used for the ANOVA groups. The results showed that firing rates of 99 neurons significantly changed around the left-moving onsets, and the numbers were 90 for the right direction, 82 for the up direction, and 85 for the down direction $(P<0.05)$. The firing rates of 71 neurons significantly changed for all four directions $(P<0.05)$, whereas 44 neurons exhibited no significant change around any type of moving direction $(P>0.05)$.

The two-sample $t$-test was used for neuronal firing windows to determine whether the window exhibited significant firing rate differences between pairwise directions (Figure 6). The grey windows in Figure 6 represented neuronal windows with significantly different firing rates between direction pairs $(P<0.05)$, whereas the black windows represented no significant change $(P>0.05)$ between direction pairs. We found that 116 neurons could be used to discriminate directions. And for every pair of directions, more than 80 neurons showed distinguishable activities, which could be used to discriminate the two directions. In addition, the same set of 54 neurons exhibited significantly different firing patterns between all direction pairs. These results demonstrated that many neurons were capable of discriminating between several direction pairs, and each direction pair could be discriminated by many neurons. Figure 6 also showed that time windows closer to moving onsets were more likely to exhibit significant firing changes. In addition, $71.59 \%$ of the discriminating windows were located between -200 and $200 \mathrm{~ms}$, which suggested that the most discriminating information was distributed around movement onsets.

\subsection{Motor direction/trajectory decoding via population level neuronal firing}

Results from the present study demonstrated that neuronal firing rates could be used for movement direction discrimination. Neuronal firing information was used to classify wrist movement directions, and a support vector machine was employed as a classifier for the study. For each neuron, only time windows before moving onsets were selected as training and testing features, because neuronal firing information would likely be used for real-time control of robot arms in the future. In total, 1490 (149 neurons $\times 10$ bins) bins were collected as classification features. Five-fold cross validation was used to obtain classification accuracy, and results revealed $96.1 \%$ classification accuracy for discriminating between four directions within 10 bins (from -1000 to $0 \mathrm{~ms}$ ). To determine temporal characteristics of neuronal firing that contributed to direction classification, less input features were incorporated into the testing and only a portion of 1490 bins were included. Classification accuracy was determined as $95.75 \%$ when bins between -1000 and $-100 \mathrm{~ms}$ were used, and accuracy values were $96.63 \%, 95.39 \%$, and $93.97 \%$ when bins were between -1000 and $-200 \mathrm{~ms},-1000$ and $-300 \mathrm{~ms}$, and -1000 and $-400 \mathrm{~ms}$, respectively. Results showed that the accuracy remained high, and most directions were discriminated when firing information from $400 \mathrm{~ms}$ before moving onsets was included.

Classification results showed that population level neuronal firing rates were able to discriminate and predict
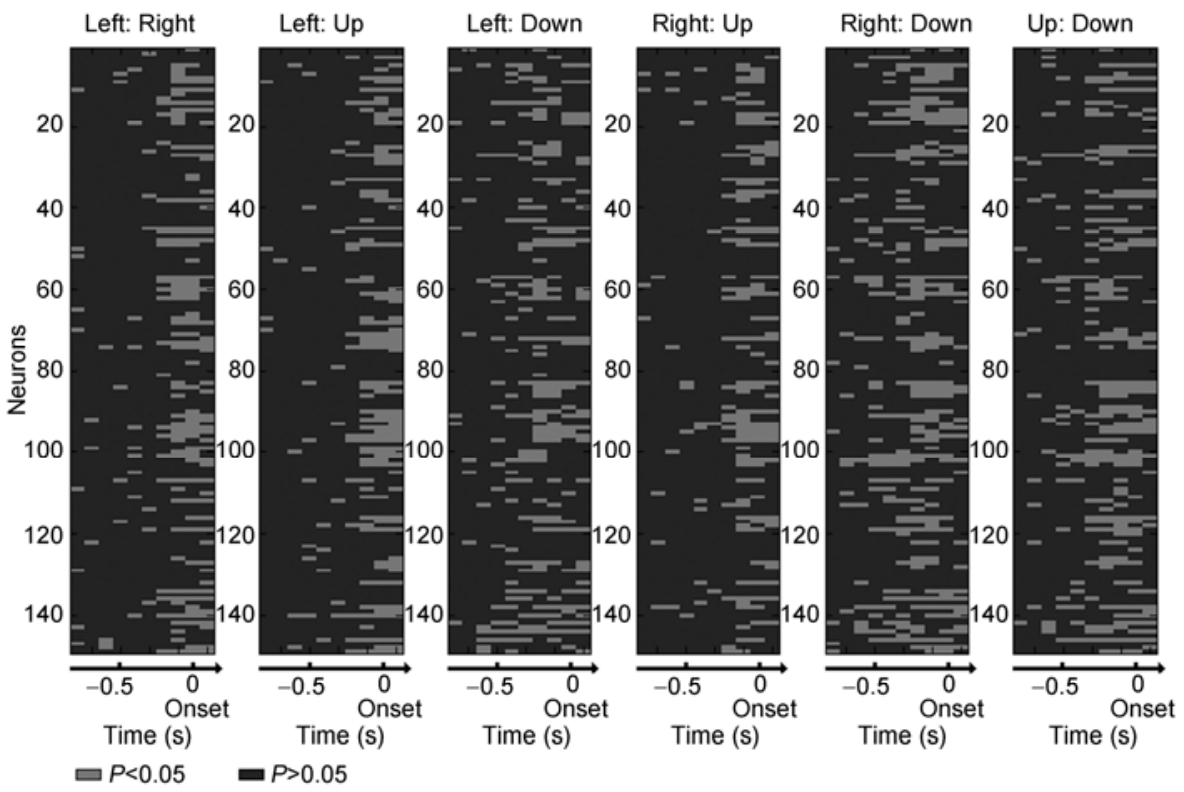

Figure 6 Significantly different neuronal firing windows between joystick movement events. Grey windows showed significant firing changes between directional joystick movement, whereas black windows showed no significant firing changes. Results were obtained using two-sample $t$-test for pairwise directional movement trials. 
monkey wrist movement directions. In addition, the BMI system has been primarily used to continuously control robotic arms or computer cursors [24,27], which has been necessary for reconstructing movement function for amputation patients rather than category classification tasks. So, neuronal firing rates were also decoded as two-dimensional continuous wrist moving trajectory with GRNN method in this study. Data recorded from monkey B01 in the afternoon of November 19, 2010 for $60 \mathrm{~min}$ served as an example. To verify neural decoding accuracy, the dataset was separated into six segments $(6 \times 10 \mathrm{~min})$. For each 10 -min data segments, one 5-min dataset served as training data for GRNN and the remaining 5-min dataset was used for testing. For each neuron, neuronal firing rates were smoothed by averaging data from ten bins (one current and nine previous bins).

Decoding performance was evaluated by Pearson's correlation coefficient (CC) and mean square error (MSE). The joystick positions along $x$-axis (Figure $7(\mathrm{a})$ ) and $y$-axis
(Figure 7(b)) were trained and tested separately. Figure 7(c) illustrated actual and estimated joystick positions from the first $11 \mathrm{~s}$ in Figure 7(a) and (b) in a two-dimensional plane. Results showed that two-dimensional joystick positions were accurately estimated by neuronal firing rates. In the first data segment, CCs were 0.8657 and 0.8242 for the $x$ - and $y$-axes, respectively. In later segments (segment 2-6), the decoding accuracy gradually decreased. The CCs decreased from 0.8 to 0.6 , and MSEs increased from 0.5 to 0.8 . These results could be a result of poor concentration compared to results from the first segment, where the monkey was more motivated for water awards. Similarly, continuous decoding results were tested using less neuronal firing information, as shown in Figure 7(d). For both $x$ - and $y$-axis positions, decoding adopted firing information between 0 and $-1000 \mathrm{~ms}$ resulted in the highest CCs and lowest MSEs, and accuracy gradually decreased when less firing information was included.
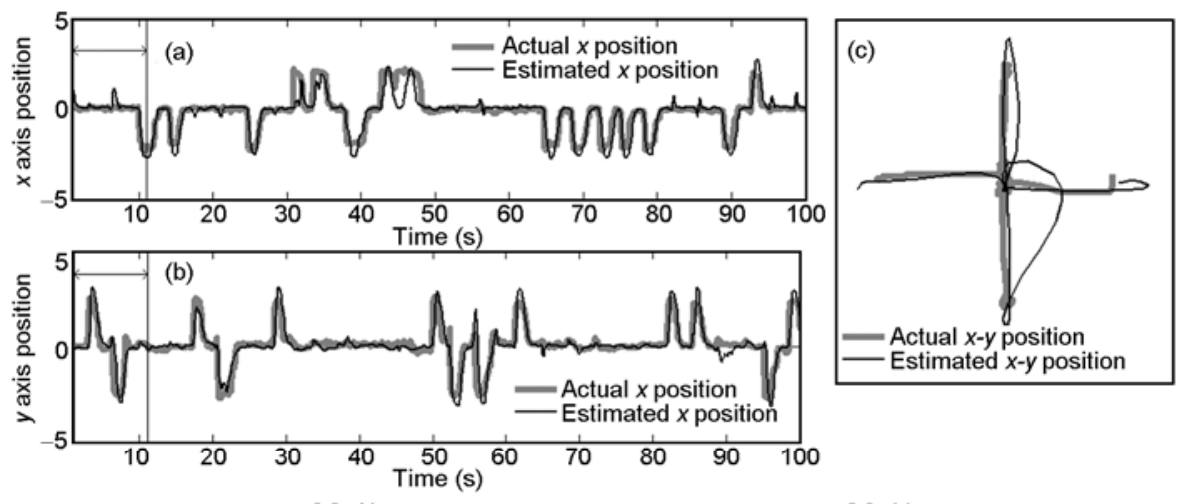

(d)
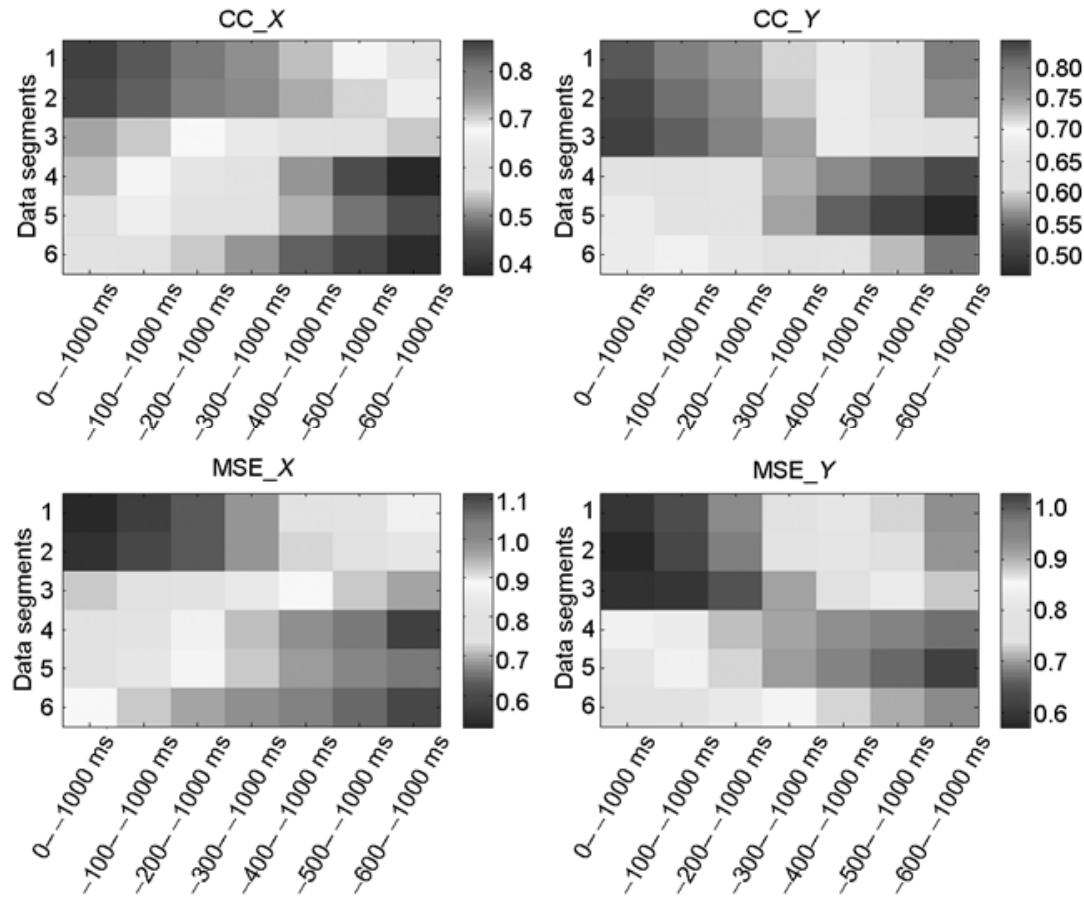

Figure 7 Motor trajectory decoding via population-level neuronal firing. (a) and (b) Illustrated actual and estimated $x$-axis and $y$-axis values, respectively, and (c) plotted decoding results in a two-dimensional plane. Grey lines indicated actual joystick positions, and dark lines indicated estimated values. (d) Illustrated continuous neural decoding accuracy via data segments and range of neural input length. 


\section{Discussion and conclusion}

Our study developed a motor-related invasive BMI system with a monkey model. Briefly, monkeys were trained to operate joystick to perform four direction center-out experiments. A 100-electrode Utah array implanted into the primary motor cortex was used to record and extract neuronal firings patterns in population level. The joystick positions were synchronously recorded with neural activities. The results demonstrated that wrist movement parameters could be predicted from neuronal activities generated by the primary motor cortex of monkey.

Previous studies have shown that neuronal activities in the primary motor cortex are related to the direction and speed of limb movements $[49,50]$. The firing rate of a neuron can be described by a cosine function of the angle between the actual moving direction and the preferred direction of the neuron. Neuronal firing with lags, or temporal information, has also been shown to correlate with limb movement [51]. Results from the present study showed similar phenomenon that neuronal firing rates highly correlated with wrist directional movement, as determined by values collected from 149 neurons at B01.

Multi-trial analysis revealed that 105 neurons exhibited significant neuronal firing changes from the baseline during directional movement. The results also showed that 71 neu- rons exhibited significant different firing patterns around the movement onsets of all four directions. For these neurons, a large amount of information from their firing patterns could be used to discriminate among moving directions (Figure 8). First, neuronal firing rates varied among directions. For example, N25 exhibited a higher firing rate for right-moving events, compared with other directions, and also exhibited lower firing rates for up-moving trials. In a similar way, N49 fired at different rates for different directions with up, right, down and left in the descending order. Second, time delays between neuron firing onset and moving onset varied among directions in some neurons, which implied that neurons could possess varying functions during directional moving onsets. As far as N27 and N84 were concerned, down-moving events resulted in quicker firing responses compared with other direction movements. It might infer that N27 could represent planning for down-moving events and execution for other directions. While N84 could execute related firing for down-moving events and exhibit sensory-related firing for other directions [8]. In addition, although most neurons from B01 showed similar firing patterns in all four directions where firing rates uniformly increased (N25) or decreased (N27 and N134) around movement onsets, some neurons did not. For $\mathrm{N} 45$, the firing rate was greater than the baseline during left-moving trials, whereas the firing rate was lower than the
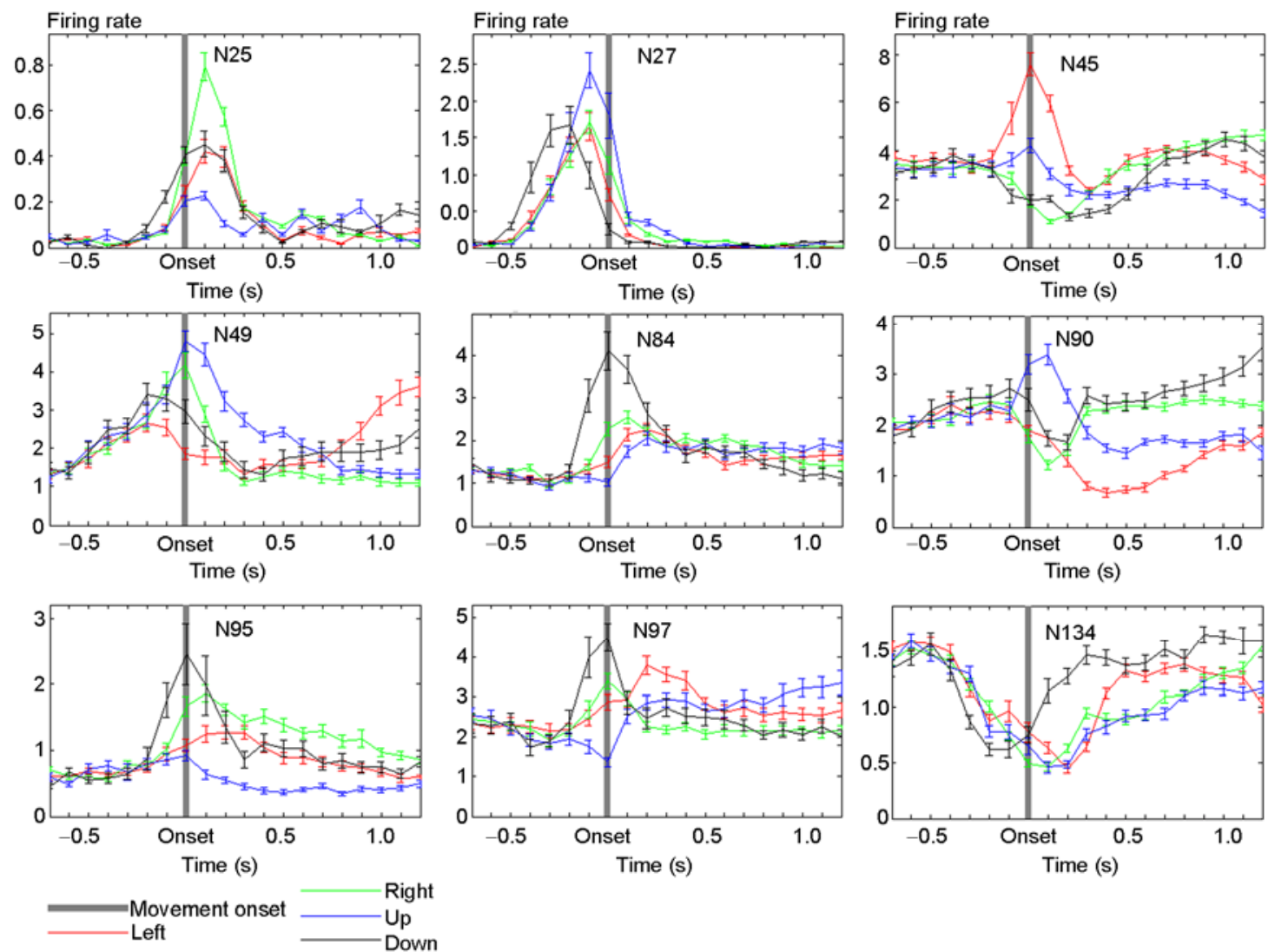

Figure 8 Neuronal firing patterns around movement onsets. Directions were indicated by different line colors, and lengths of short bars represented standard deviation of trials divided by root of trial number. Grey lines denoted onset of movement trials. 
baseline during right and down-moving trials. The similar firing pattern was found in $\mathrm{N} 90$ where up-moving trials exhibited higher firing rates than at baseline and the firing rates were less than at baseline in other moving directions. All firing differences in either amplitude or phase could help to discriminate different movement directions. Results in Figure 6 demonstrated that most discriminating information was located between -200 and $200 \mathrm{~ms}$.

It has been previously reported that the neuronal activities concerning movement execution happened at 200-300 ms before movement onset [37]. The role of neuronal activities from -1000 to $-400 \mathrm{~ms}$ before movement onset was evaluated in this study because it was found that many neurons exhibited very early firing prior to movement onsets. N27 and N134 initiated response-movement events at 400-500 ms prior to onset. For SVM classification of movement direction, when neuronal firing activities between -100 and 0 ms were adopted, the accuracy of classification is $95.75 \%$. However, the result was kept in $93.97 \%$ when neuronal firing activities between -1000 and $0 \mathrm{~ms}$ were applied. It was suggested that many of the firing windows prior to $-200 \mathrm{~ms}$ also could be used to discriminate moving direction. In addition, for continuous regression, the decoding accuracies were 0.719 and 0.671 for $x$ - and $y$-axis values respectively when neuronal firing activities from -1000 to $-400 \mathrm{~ms}$ were applied. Although the results could still reflect movement trends, it was suggested that these segments of neuronal activities did not contain enough information about the movement when they were used to decode the trajectory of monkey's wrist movement. Early neuronal firing activities $(-400 \mathrm{~ms})$ could be related to a response of visual stimulation, but not motor execution. The center-out experiment used in this study was a visually guided motor task where the movement of monkey's wrist was guided by a visual cue. The time from appearance of the target to moving onset was found to be $400-520 \mathrm{~ms}$. Therefore the motor cortex might indirectly receive the afferent signals from visual neural pathway and change its firing pattern. The physiological origination and function of the early neuronal firing activity required further studies, because this study primarily described the relationship between neuronal activities and movement parameters but not focus on other issues, such as the integration of visual signals into motor behavior.

Novel developments in BMI systems have depended on the advantage of chronically implantable microelectrode arrays and neural decoding algorithms. To date, most neural decoding methods were divided into single-stage or two-stage. One-stage methods have been used to directly estimate movement parameters using linear or non-linear models, such as linear regression, artificial neural networks and Bayesian filters. Two-stage neural decoding methods, however, have typically taken into account the dividing and coordinating mechanisms of neural information processing that connect biological movement stages and neuronal firing patterns. The two-stage decoding algorithms are implemented using different decoding models in different movement stages [52]. Although two-stage methods can reflect the neural activities even more, they might also require prior knowledge and training for locating and constructing specific decoding models. Therefore, the single-stage decoding method has been more popular because of application convenience. In this study, classification and regression were realized by using single-stage models, SVM and GRNN, respectively. Although the two decoding algorithms in our BMI system performed with high neural decoding accuracy in discrete direction classification and continuous trajectory decoding, they had to use the nearest training data and results were corrupt if only the first training segment was adopted. Further studies were needed to better understand the physiological mechanisms of brain expression about limb movement, which would improve the performance of BMI system with some adaptive neural decoding algorithms. In addition, an applied BMI system should use external actuator control strategies to achieve real-time control. One potential solution for this challenge is to implement decoding algorithms in parallel computing processors such as a field-programmable gate array (FPGA) [53].

This work was supported by the National Natural Science Foundation of China (61031002, 61001172), the National Basic Research Program of China (2011CB504405), the Zhejiang Provincial Key Science and Technology Program for International Cooperation (2011C14005). We cordially and sincerely thank Professor Wu Li from Beijing Normal University for sharing valuable experience of monkey training and array implantation surgery, Dr. Paul House from University of Utah and Dr. Shaohua Xи from State University of New York for valuable surgery instructions.

1 Gilja V, Chestek C A, Diester I, et al. Challenges and opportunities for next-generation intracortically based neural prostheses. IEEE Trans Biomed Eng, 2011, 58: 1891-1899

2 Vaadia E, Birbaumer N. Grand challenges of brain computer interfaces in the years to come. Front Neurosci, 2009, 3: 151-154

3 Hatsopoulos N G, Donoghue J P. The science of neural interface systems. Annu Rev Neurosci, 2009, 32: 249-266

4 Schwartz A B, Cui X T, Weber D J, et al. Brain-controlled interfaces: Movement restoration with neural prosthetics. Neuron, 2006, 52: 205-220

5 Lebedev M A, Nicolelis M A. Brain-machine interfaces: Past, present and future. Trends Neurosci, 2006, 29: 536-546

6 Nicolelis M A. Actions from thoughts. Nature, 2001, 409: 403-407

7 Donoghue J P. Bridging the brain to the world: A perspective on neural interface systems. Neuron, 2008, 60: 511-521

8 Chapin J K, Moxon K A, Markowitz R S, et al. Real-time control of a robot arm using simultaneously recorded neurons in the motor cortex. Nat Neurosci, 1999, 2: 664-670

9 Hochberg L R, Donoghue J P. Sensors for brain-computer interfaces. IEEE Eng Med Biol Mag, 2006, 25: 32-38

10 Watanabe $\mathrm{H}$, Takahashi $\mathrm{H}$, Nakao $\mathrm{M}$, et al. Intravascular neural interface with nanowire electrode. Electron Commun Jpn, 2009, 92: 29-37

11 Shenoy K V, Chestek C A, Gilja V, et al. Long-term stability of neural prosthetic control signals from silicon cortical arrays in rhesus macaque motor cortex. J Neural Eng, 2011, 8: 1-11

12 Stieglitz T, Rubehn B, Henle C, et al. Brain-computer interfaces: An overview of the hardware to record neural signals from the cortex. 
Prog Brain Res, 2009, 175: 297-315

13 Quiroga R Q, Panzeri S. Extracting information from neuronal populations: Information theory and decoding approaches. Nat Rev Neurosci, 2009, 10: 173-185

14 Gupta R, Ashe J. Offline decoding of end-point forces using neural ensembles: Application to a brain-machine interface. IEEE Trans Neural Syst Rehabil Eng, 2009, 17: 254-262

15 Milan R J, Carmena J M. Invasive or noninvasive: Understanding brain-machine interface technology. IEEE Eng Med Biol Mag, 2010, 29: $16-22$

16 Leeb R, Sagha H, Chavarriaga R, et al. A hybrid brain-computer interface based on the fusion of electroencephalographic and electromyographic activities. J Neural Eng, 2011, 8: 1-5

17 Zhao Q B, Zhang L Q, Cichocki A. EEG-based asynchronous BCI control of a car in 3D virtual reality environments. Chin Sci Bull, 2009, 54: 78-87

18 Long J, Li Y, Yu T, et al. Target selection with hybrid feature for BCI-based 2-D cursor control. IEEE Trans Biomed Eng, 2011, 14: 1-8

19 Hammon P, Makeig S, Poizner H, et al. Predicting reaching targets from human EEG. IEEE Signal Process Mag, 2008, 25: 69-77

20 Stevenson I H, Cherian A, London B M, et al. Statistical assessment of the stability of neural movement representations. J Neurophysiol, 2011, 106: 764-774

21 Ince N F, Gupta R, Arica S, et al. High accuracy decoding of movement target direction in non-human primates based on common spatial patterns of local field potentials. PLoS ONE, 2010, 5: 1-11

22 Zhang S, Jiang B, Zhu J, et al. A study on combining local field potential and single unit activity for better neural decoding. Int J Imag Syst Tech, 2011, 21: 165-172

23 Yu Y, Zhang S M, Zhang H J, et al. Neural decoding based on probabilistic neural network. J Zhejiang Univ Sci B, 2010, 11: 298-306

24 Velliste M, Perel S, Spalding M C, et al. Cortical control of a prosthetic arm for self-feeding. Nature, 2008, 453: 1098-1101

25 Moritz C T, Perlmutter S I, Fetz E E. Direct control of paralysed muscles by cortical neurons. Nature, 2008, 456: 639-642

26 Wessberg J, Stambaugh C R, Kralik J D, et al. Real-time prediction of hand trajectory by ensembles of cortical neurons in primates. Nature, 2000, 408: 361-365

27 Serruya M D, Hatsopoulos N G, Paninski L, et al. Instant neural control of a movement signal. Nature, 2002, 416: 141-142

28 Simeral J D, Kim S P, Black M J, et al. Neural control of cursor trajectory and click by a human with tetraplegia 1000 days after implant of an intracortical microelectrode array. J Neural Eng, 2011, 8: 1-24

29 Orban G A, Van E D, Vanduffel W. Comparative mapping of higher visual areas in monkeys and humans. Trends Cognit Sci, 2004, 8: 315-324

30 Carpaneto J, Umiltà M A, Fogassi L, et al. Decoding the activity of grasping neurons recorded from the ventral premotor area F5 of the macaque monkey. Neuroscience, 2011, 188: 80-94

31 Nicolelis M A, Dimitrov D, Carmena J M, et al. Chronic, multisite, multielectrode recordings in macaque monkeys. Proc Natl Acad Sci USA, 2003, 100: 11041-11046

32 Hendrix C M, Mason C R, Ebner T J. Signaling of grasp dimension and grasp force in dorsal premotor cortex and primary motor cortex neurons during reach to grasp in the monkey. J Neurophysiol, 2009, 102: $132-145$
33 Green A M, Kalaska J F. Learning to move machines with the mind. Trends Neurosci, 2011, 34: 61-75

34 Georgopoulos A P, Schwartz A B, Kettner R E. Neuronal population coding of movement direction. Science, 1986, 233: 1416-1419

35 Georgopoulos A P, Kalaska J F, Caminiti R, et al. On the relations between the direction of two-dimensional arm movements and cell discharge in primate motor cortex. J Neurosci, 1982, 2: 1527-1537

36 Ganguly K, Secundo L, Ranade G, et al. Cortical representation of ipsilateral arm movements in monkey and man. J Neurosci, 2009, 29: 12948-12956

37 Kemere C, Santhanam G, Yu B M, et al. Detecting neural-state transitions using hidden Markov models for motor cortical prostheses. J Neurophysiol, 2008, 100: 2441-2452

38 Vargas-Irwin C E, Shakhnarovich G, Yadollahpour P, et al. Decoding complete reach and grasp actions from local primary motor cortex populations. J Neurosci, 2010, 30: 9659-9669

39 Taylor D M, Helms S I, Schwartz A B. Direct cortical control of 3D neuroprosthetic devices. Science, 2002, 296: 1829-1832

40 Acharya S, Tenore F, Aggarwal V, et al. Decoding individuated finger movements using volume-constrained neuronal ensembles in the M1 hand area. IEEE Trans Neural Syst Rehabil Eng, 2008, 16: 15-23

41 Olson B P, Si J, Hu J, et al. Closed-loop cortical control of direction using support vector machines. IEEE Trans Neural Syst Rehabil Eng, 2005, 13: 72-80

42 Stark E, Abeles M. Predicting movement from multiunit activity. J Neurosci, 2007, 27: 8387-8394

43 Zhang Q, Zhang S, Lin J, et al. Building brain machine interfaces: From rat to monkey. In: Yen J Y, Hsu P L, Tsai S H, eds. Proceedings of 2011 8th Asian Control Conference, 2011 May 15-18, Kaohsiung. California: IEEE Computer Society, 2011. 886-891

44 Suner S, Fellows M R, Vargas-Irwin C, et al. Reliability of signals from a chronically implanted, silicon-based electrode array in non-human primate primary motor cortex. IEEE Trans Neural Syst Rehabil Eng, 2005, 13: 524-541

45 Meyer D, Leisch F, Hornik K. The support vector machine under test. Neurocomputing, 2003, 55: 169-186

46 Specht D F. A general regression neural network. IEEE Trans Neural Netw, 1991, 2: 568-576

47 Parzen E. On estimation of a probability density function and mode. Ann Math Stat, 1962, 33: 1065-1076

48 Chestek C A, Batista A P, Santhanam G, et al. Single-neuron stability during repeated reaching in macaque premotor cortex. J Neurosci, 2007, 27: 10742-10750

49 Amirikian B, Georgopulos A P. Directional tuning profiles of motor cortical cells. Neurosci Res, 2000, 36: 73-79

50 Fitzsimmons N A, Lebedev M A, Peikon I D, et al. Extracting kinematic parameters for monkey bipedal walking from cortical neuronal ensemble activity. Front Integr Neurosci, 2009, 3: 1-19

51 Lebedev M A, Carmena J M, O'Doherty J E, et al. Cortical ensemble adaptation to represent velocity of an artificial actuator controlled by a brain-machine interface. J Neurosci, 2005, 25: 4681-4693

52 Ma W J, Beck J M, Latham P E, et al. Bayesian inference with probabilistic population codes. Nat Neurosci, 2006, 9: 1432-1438

53 Zhou F, Liu J, Yu Y, et al. Field-programmable gate array implementation of a probabilistic neural network for motor cortical decoding in rats. J Neurosci Methods, 2010, 185: 299-306

Open Access This article is distributed under the terms of the Creative Commons Attribution License which permits any use, distribution, and reproduction in any medium, provided the original author(s) and source are credited. 